\title{
Re-Thinking Justice for Janitors - Strategische Erneuerung der amerikanischen Dienstleistungsgewerkschaft SEIU
}

Keine andere Kampagne symbolisiert das bewegungsorientierte Organizing-Modell und die Revitalisierung der amerikanischen Gewerkschaften so sehr wie die Justice for Janitors-Kampagne der Dienstleistungsgewerkschaft Service Employees International Union (SEIU). Mit einer konsequenten Wachstumsstrategie und zahlreichen umfassenden Organizing-Kampagnen, die derzeit auch verstärktes Interesse bei deutschen Gewerkschaften finden, gelang es der SEIU von 1990 bis 2006 ihre Mitgliederzahlen zu verdoppeln und zur größten Gewerkschaft der USA zu werden. Nun strebt die SEIU eine Ausweitung des bewährten Organisierungsmodells auf bislang unerschlossene Branchen und Regionen an. Dieses Vorhaben wirft die Frage auf, inwieweit sich gewerkschaftliches Wachstum planen und organisieren lässt und wie die SEIU dabei vorgeht.

\section{Einleitung}

Seit geraumer Zeit beschäftigt sich die gewerkschaftssoziologische Forschung, insbesondere im angelsächsischen Sprachraum, verstärkt mit Erneuerungsansätzen von Gewerkschaften in der Krise (Frege/ Kelly 2004). Die Revitalisierung einiger Gewerkschaften wurde in diesem Zusammenhang als Beleg dafür interpretiert, dass Gewerkschaften mitnichten lediglich Getriebene des globalisierten Kapitalismus seien, sondern durchaus in der Lage wären, pro-aktiv auf die Krise zu reagieren (Frege/ Kelly 2004; Hurd et al. 2003; Turner et al. 2001). Insbesondere innovative Strategien der US-amerikanischen Gewerkschaften zur Überwindung der Mitgliederkrise, gemeinhin als „Organizing“ bezeichnet (Dribbusch 2007), nehmen dabei eine prominente Rolle ein. Die Entscheidung einiger amerikanischer Gewerkschaften Mitte der 1990er Jahre, angesichts des dramatischen Rückgangs des Organisationsgrads tief greifende Strukturreformen durchzusetzen, wurde in der wissenschaftlichen Literatur ausgiebig diskutiert und bescherte nicht zuletzt der US-Gewerkschaftsforschung eine Renaissance (Labor Revitalization Studies). ${ }^{1}$

Speziell die amerikanische Dienstleistungsgewerkschaft Service Employees International Union (SEIU) wird aufmerksam beobachtet und gilt als besonders innovativ. Sie hat für sich seit vielen Jahren die Erweiterung der Organisation zum zentralen strategischen Ziel erklärt. Die SEIU hat seit
1990 ihre Mitgliederzahl in den USA, Kanada und Puerto Rico auf 1,9 Mio. verdoppelt und ist die größte und am schnellsten wachsende Gewerkschaft in den USA. ${ }^{2}$ Am bekanntesten ist ihre Kampagne zur Organisierung von Reinigungskräften: Justice for Janitors (dt.: Gerechtigkeit für Reinigungskräfte).

Justice for Janitors (JfJ) hat in vielerlei Hinsicht eine Pionierrolle für die Revitalisierung der amerikanischen Gewerkschaftsbewegung eingenommen und erfüllt diese Funktion nach wie vor. Derzeit praktiziert und evaluiert die SEIU Expansionsstrategien von JfJ, die im vorliegenden Beitrag vorgestellt werden. Zunächst jedoch wird ein Überblick über die Kampagne und ihre Bedeutung für den Erneuerungsprozess der amerikanischen Gewerkschaften und der SEIU gegeben.

Die folgende Analyse basiert auf eigenen, im Jahr 2007 durchgeführten Studien bei verschiedenen Locals der SEIU sowie einer Auswertung von Gewerkschaftsunterlagen. Der Fokus der Untersuchung liegt auf der Property Services Division der SEIU, der mit 225.000 Mitgliedern größten Gewerkschaftsorganisation im Bereich Gebäudedienstleistungen in den USA. ${ }^{3}$

\section{Das Prinzip Justice for Janitors: Strategie und Taktik}

Justice for Janitors (JfJ) wurde in den 1980er Jahren von der SEIU-Führung als Reaktion auf dramatische Mitgliederverluste durch
Outsourcing der Gebäudedienstleistungen im kommerziellen Immobiliensektor initiiert. Die SEIU entwickelte eine Strategie, die darauf abzielte, den gesamten Markt für Reinigungsdienste einer Stadt zu kontrollieren und Flächenstandards durchzusetzen. Dies stellte für die USA insofern einen innovativen Ansatz dar, als hier gewerkschaftliche Interessenvertretung üblicherweise Betrieb für Betrieb in einem komplizierten Anerkennungsverfahren durchgesetzt werden muss (Fantasia/Voss 2004;

\footnotetext{
1 Die derzeitig zunehmende Literatur umfasst unter anderem: Bronfenbrenner et al. 1998; Fairbrother/ Yates 2003; Fantasia/Voss 2004; Heery 2003; Milkman/Voss 2004; Turner et al. 2001; Turner/ Cornfield 2007; Überblick im deutschsprachigen Raum: Brinkmann et al. 2008.

2 Vgl. SEIU Website: http://www.seiu.org/about/ index.cfm, Download vom 30.10.2007

3 Die anderen Divisions (Abteilungen) sind Healthcare Division (Gesundheitswesen), unterteilt in Hospital und Homecare Workers mit 900.000 Mitgliedern, und die Public Service Division (öffentlicher Dienst) mit 850.000 Mitgliedern (alle Zahlen 2007). Damit ist die SEIU die größte Gewerkschaft im Gesundheitswesen und die zweitgrößte im öffentlichen Dienst. Die Divisions genießen eine hohe Autonomie und arbeiten sehr unterschiedlich.
}

Hae-Lin Choi, Politikwissenschaftlerin, promoviert an der Freien Universität Berlin, Fachbereich Politikwissenschaft, über gewerkschaftliche Organisierungsstrategien in den USA, Südkorea und Italien. Arbeitsschwerpunkte: Prekäre Beschäftigung, gewerkschaftliche Mitgliedergewinnung und gewerkschaftliche Revitalisierungsstrategien. e-mail: haelin.choi@gmail.com 
Katz/Kochan 2003). ${ }^{4}$ Seit Mitte der 1980er Jahre haben sich einige reformorientierte Gewerkschaften, ${ }^{5}$ zunehmend darauf konzentriert, die Arbeitgeber ohne formelle Anerkennungswahlen mit aggressiven Kampagnen zu Neutralitätsabkommen, sogenannte Card Check Neutrality Agreements, zu zwingen. Hierfür wird, wie im Fall von JfJ, Druck auf führende Auftraggeber, in der Regel Immobilienbesitzer, ausgeübt, um ein Abkommen (Master Agreement) mit der Gewerkschaft über die ausschließliche Nutzung von gewerkschaftlichen Reinigungsfirmen abzuschließen, sodass die Löhne nicht mehr Teil des Wettbewerbs sind. Dann wird Druck auf die Arbeitgeber ausgeübt, einen Tarifvertrag (Master Contract) mit der Gewerkschaft abzuschließen, der jedoch erst dann in Kraft tritt, wenn mindestens $65 \%$ der marktführenden Reinigungsfirmen organisiert sind. So werden bereits organisierte Unternehmen geschützt und noch zu organisierende Unternehmen haben einen Anreiz zur Unterzeichnung (trigger effect) (Casey-Bolaños 1999).

\subsection{KAMPAGNENFORMEN}

Gewerkschaftlicher Druck wird üblicherweise entweder anhand von Unternehmenskampagnen (Corporate Campaigns) oder umfassenden Kampagnen (Comprehensive Campaigns) aufgebaut. Corporate Campaigns haben mit wachsendem Unternehmenswiderstand gegen gewerkschaftliche Organisierungsversuche an Bedeutung gewonnen. Sie zielen darauf ab, gewerkschaftsfeindlichen Unternehmen systematisch Schaden zuzufügen oder sie sogar aus dem Markt zu drängen, was insbesondere bei Subunternehmen angewandt wird, um gewerkschaftlichen Vertragsnehmern Marktvorteile zu verschaffen (Choi 2008; Manheim 2005). Corporate campaigns werden in der Regel von Hauptamtlichen durchgeführt (staff driven) und bedürfen oft nur geringer oder keiner Beteiligung der Beschäftigten. Dies konfligiert tendenziell mit den stark auf Basisaktivierung ausgerichteten Grundgedanken des Organizing Model (Bronfenbrenner/Hickey 2004, S. 19). In umfassenden Kampagnen ist die Beteiligung und Mobilisierung der Beschäftigten hingegen weitaus zentraler. Hierbei wird der Druck auf die Zielunternehmen in inner- und außerbetrieblichen Aktionen auf mehreren Ebenen aufgebaut und von den Beschäftigten, der Gewerkschaft und anderen zivilgesellschaftlichen
Akteuren getragen. Im Vergleich mit Unternehmenskampagnen sind umfassende Kampagnen weitaus komplexer, ressourcenintensiver und langwieriger.

\subsection{SOCIAL JUSTICE UNIONISM}

Justice for Janitors ist zum Paradebeispiel einer Comprehensive Campaign geworden. Sie steht für die mit dem Begriff Organizing verbundenen innovativen Organisierungstechniken (Bronfenbrenner/Hickey 2004), Koalitionen mit lokalen Gemeindeorganisationen, sozialen Bewegungen und Kirchen und militante Aktionsformen, mit denen Druck auf die Arbeitgeber ausgeübt wird. JfJ brachte der SEIU das Image einer bewegungsorientierten "Social Justice Union"6 ein (Dribbusch 1998; Fantasia/ Voss 2004, S. 134). Dieser Ansatz ist jedoch nicht mit partizipatorischer Gewerkschaftsdemokratie und bottom-up-Ansätzen, die andere Organisierungsmodelle charakterisieren, zu verwechseln. JfJ war von Beginn an eine strategische, zentral organisierte und durchgeführte Mitgliedergewinnungskampagne. Milkman/Wong (2001) und Savage (2006) haben auf die top-down Elemente von Jff hingewiesen, die diese Kampagne nach wie vor auszeichnen. Doch das wohl wesentlichste Merkmal der Kampagne war die erstmalig breite Organisierung und Sichtbarmachung von migrantischen Beschäftigten im Niedriglohnsektor, die bis dato als „unorganisierbar" galten (Erickson et al.2007). ${ }^{7}$ Dies war jedoch keine bewusste Entscheidung der SEIU, sondern resultierte aus der Prekarisierung in diesem Sektor und der Veränderung der Beschäftigtenstruktur, der sich die SEIU anpassen musste. Doch JfJ hat gezeigt, dass eine Organisierung der vermeintlich Unorganisierbaren möglich ist. Inzwischen gelten Migranten im Niedriglohnsektor als Beschäftigte mit den stärksten Organisierungsneigungen (Milkman 2006b).

Die SEIU hat mit Justice for Janitors innerhalb von zwei Dekaden den Reinigungssektor im Bereich kommerzieller Immobilien zurückgewonnen und 225.000 Reinigungskräfte organisiert. Sie kann mittlerweile 29 Master Contracts vorweisen und erzielt Organisationsgrade von $65 \%$ bis $90 \%$ in zentralen urbanen und vorstädtischen Regionen in den USA (SEIU 2007). Doch vor allem hat JfJ der SEIU und der gesamten Gewerkschaftsbewegung bewiesen, dass es auch unter widrigsten Umständen möglich ist, gewerkschaftliche Kämpfe zu gewinnen.

\section{Die Erneuerung der SEIU zur Organizing-Union}

Die Erfolgsgeschichte von JfJ ist eingebettet in die generelle Transformation der SEIU zu einer Organisierungsgewerkschaft (Organizing Union) (Fletcher/Hurd 1998). Andy Stern, der Nachfolger John Sweeneys als Präsident der SEIU, führte dessen Reformkurs fort und verordnete der Gewerkschaft grundlegende Restrukturierungen und Zentralisierungen (Stern 2004, S. 55). Auf ihren Gewerkschaftskongressen 2000 und 2004 verabschiedete die SEIU radikale Reformpläne mit programmatischen Titeln wie New Strength Unity Plan (2000) und Seven Strengths (2004), die allen lokalen Gewerkschaftseinheiten, die sogenannten Locals, eine jährliche Wachstumsrate von $5 \%$ vorgaben, wobei mindestens $20 \%$ des Budgets für Mitgliedergewinnung verwendet werden sollte (Long 2007). ${ }^{8}$

4 In einem ersten Schritt müssen sich $30 \%$ der Belegschaft für Anerkennungswahlen aussprechen und dann leitet das National Labor Relations Board (NLRB) offiziell Wahlen ein. Danach muss die Gewerkschaft von über $50 \%$ der Belegschaft gewählt werden, um offiziell das Vertretungsrecht zu erlangen. Diese Prozedur ist langwierig und mit zahlreichen Schwierigkeiten verbunden, da die Arbeitgeber in der Regel die Wahlen behindern, oftmals mit Unterstützung von professionellen Unternehmensberatungsagenturen, die sich auf Gewerkschaftsabwehr spezialisiert haben (Bronfenbrenner 1994; Levine 2007; Logan 2006).

5 Das sind neben der SEIU vor allem die Union of Needletrades, Industrial \& Textile Employees Hotel Employees \& Restaurant Employees International Union (UNITE-HERE), die Communications Workers of America (CWA) und die United Food and Commercial Workers International Union (UFCW).

6 Am ehesten mit "sozialer Bewegungsgewerkschaft" zu übersetzen.

7 Das politische Engagement der SEIU im Bereich Immigrationspolitik hat ihr eine respektierte Stellung innerhalb von migrantischen Gemeinschaften eingebracht und genießt besondere Priorität, was sicherlich auf den Einfluss der Mitglieder zurückzuführen ist.

8 Die SEIU forderte darüber hinaus auch von anderen Gewerkschaften ähnliche Maßnahmen zur Bekämpfung der Mitgliederverluste. Diese Kontroverse mündete in der Abspaltung der eher reformorienterten Gewerkschaften vom Dachverband und zur Gründung der Change-To-Win Koalition, deren acht Gewerkschaften nun mit rund 6 Mio. Mitgliedern fast so viele Beschäftigte vertreten, wie die restlichen 60 Gewerkschaften, die im AFL-CIO verblieben sind (Greer 2006; Knowles 2007) 
Um die massenhafte Organisierung zu finanzieren, wurden mit Beschluss des Gewerkschaftskongresses von 2000 die Mitgliederbeiträge der SEIU um einen Dollar pro Woche erhöht und ein Fonds zur Finanzierung von innovativen DurchbruchsKampagnen eingerichtet (Unity Funds) (SEIU 2003). Durchbruchs-Kampagnen dienen der strategischen Erschließung von neuen Branchen oder Regionen, in denen gewerkschaftliche Strukturen grundlegend neu gebildet werden müssen. Ein weiterer Schritt zur Einbindung der Mitglieder in den Organisierungsprozess ist die Einrichtung von sogenannten „Mitglieder-Brigaden" (Member Brigades), die für Aktionen, Demonstrationen und zur Streikunterstützung (Honorary Picket Lines) von der Gewerkschaft engagiert und bezahlt werden und für diese Zeit von ihrer eigentlichen Tätigkeit beurlaubt sind. ${ }^{9}$ Diese Brigaden nehmen auch verstärkt ehrenamtliche oder hauptamtliche Funktionen als Organizer ein, da sie aufgrund ihrer Authentizität die besten Organisierungsresultate erzielen.

Eine zweite bedeutende Änderung war die Zentralisierung der Gewerkschaft. Nach Ansicht der SEIU-Führung sind größere Locals mit mehr Ressourcen und Verhandlungsmacht besser in der Lage, groß angelegte überregionale Kampagnen durchzuführen (Lerner 2001). Zahlreiche städtische Gewerkschaftseinheiten wurden $\mathrm{zu}$ regionalen Locals zusammengelegt. Locals, die sich der Ausrichtung auf Mitgliedergewinnung verweigerten, wurden direkt der Zentrale unterstellt (trusteeship). Ein Prozess, der als undemokratisch kritisiert wurde und von vielen Widerständen begleitet war und ist, da er mit Partizipationsverlusten der Mitglieder verbunden war und der Führung der Locals Machtzuwächse brachte (Fletcher/Hurd 1998; Lopez 2004). Für den Fachbereich der Gebäudedienstleistungen bedeutete dies eine Reduzierung von 20 Locals im Jahr 2000 auf heute 11 Locals mit entsprechend großen geografischer Ausdehnung (Long 2007)10. Ein weiterer Grund für die Zentralisierung war die Bestrebung, die gewerkschaftliche Struktur den veränderten Branchenstrukturen anzupassen. Zentrales Motiv der SEIU ist der Aufbau von branchenweiter Marktmacht (Industry Power).
Kriterien, Machbarkeit und Gewinnwahrscheinlichkeit analysiert und evaluiert, damit schließlich zwei bis drei zentrale Strategien verabschiedet werden können.

\subsection{DENSITY CAMPAIGNS: $90 \%$}

Die SEIU hebt sich von vielen anderen Gewerkschaften durch die ausgesprochen strategische Planung der Mitgliedergewinnung $\mathrm{ab}$. So verfügt sie über einen wachsenden Stamm von wirtschaftlich geschulten Analysten, die Expansionsmöglichkeiten (New Growth Research) recherchieren. Für Organisierungsziele, die als vorteilhaft und erreichbar eingeschätzt werden, werden Strategien entworfen und Kampagnen entwickelt. Prinzipiell kann das Wachstum in drei Richtungen stattfinden: Schließung der Organisierungslücken in bestehenden Regionen, sogenannte Gewerkschaftsmärkte (Union Markets), Ausweitung auf neue Regionen (New Markets) und Ausweitung auf neue Branchen und Beschäftigtengruppen in Union Markets und New Markets. Diese Optionen werden derzeit in unterschiedlichen Kombinationen und Ausrichtungen getestet und diskutiert:

- Erhöhung des Organisationsgrads auf $90 \%$ in bereits organisierten Sektoren (90\% Density Campaigns),

- Ausweitung auf neue Sektoren (New Sector Campaigns),

- nationale kundenorientierte Kampagnen (National Clientbased Campaigns), - nationale arbeitgeberorientierte Kampagnen (National Contractor Campaigns), und

- Zugewinn von neuen, bislang unerschlossenen Regionen wie die südlichen Staaten der USA oder Right-to-work-Staaten (New Market Campaigns) (Long 2007; Pastreich 2007).

Bis auf die erste Strategie handelt es sich um nationale Kampagnen, bei denen verschiedene Locals kooperieren. Bereits der stadtweite Ansatz der SEIU ist für die extrem dezentral operierenden US-Gewerkschaften sehr untypisch, und mit nationalen Kampagnen gegen nationale Auftragoder Arbeitsgeber geht die SEIU noch einen Schritt weiter. Bis zum Gewerkschaftskongress 2008 werden diese Strategien vom Visioning-Committee der SEIU-Führung, besetzt mit den Fachbereichsvorsitzenden und beauftragt mit der Entwicklung von Zukunftsstrategien, nach Kosten-Nutzen
Die Erhöhung des Organisationsgrads zielt auf die Expansion, entweder in Richtung neuer Sektoren oder neuer Beschäftigtengruppen innerhalb von existierenden Union Markets ab. Neue Sektoren jenseits der klassischen Bürokomplexe umfassen zum Beispiel private Wohngebiete, den öffentlichen Sektor, Groß- und Einzelhandel, Universitäten und Bildungseinrichtungen; neue Beschäftigtengruppen hingegen beziehen sich in erster Linie auf die Organisierung von Sicherheitsleuten, die oftmals für dieselben Unternehmen arbeiten wie die Reinigungskräfte. Seit 2004 führt die SEIU in Anlehnung an JfJ eine nationale Kampagne zur Organisierung von Sicherheitskräften mit dem Namen Stand for Security $^{11}$ durch, die das derzeit größte Erweiterungsprojekt der SEIU darstellt und die Vorlage für das von ver.di in Hamburg durchgeführte Organizingprojekt bildete (Bremme et al. 2007). Der Vorteil dieser Strategie besteht darin, dass bereits aufgebaute Strukturen und deren Verbindungen und Einfluss genutzt werden können. Insgesamt geht die SEIU von einem Wachstumspotenzial von 200.000 Beschäftigten aus.

\subsection{NEW SECTOR CAMPAIGNS}

Die Ausweitung auf neue Sektoren wird derzeit im Bereich Multi-Service Unternehmen versucht und stellt eine der weitreichendsten Erneuerungen für die SEIU dar. Hierbei werden zwei Ansätze vereint: Die Ausweitung auf neue Beschäftigtengruppen und die Konzentration auf nationale Arbeitgeber.

Die Rückkehrgarantie der Brigademitglieder ist mittlerweile ein wichtiger Standardpassus in allen Tarifverträgen, die die Gewerkschaft abschließt.

10 Eine andere, vielfach kritisierte Wachstumsstrategie war die Einverleibung von externen, unabhängigen Gewerkschaften in die SEIU, was mittlerweile kaum noch praktiziert wird, da es zu keinem wirklichen Wachstum führte, sondern lediglich die SEIU stärkte (Long 2007).

11 Vgl. Website http://www.standforsecurity.org, Download vom 20.10.2007. 
Anfang 2004 ging die SEIU eine Kooperation mit der Textil-, Hotel- und Restaurantarbeitergewerkschaft UNITEHERE ein, um die bislang völlig unorganisierte Multi-Service Branche zu organisieren. ${ }^{12}$ Diese Branche umfasst große Konzerne, die ein umfassendes Serviceangebot offerieren, zu dem beispielsweise Textilreinigung, Catering und Instandhaltung gehören. In den USA dominieren derzeit drei multinationale Unternehmen, die „Big 3“ (Aramark, Compass, Sodexho), diesen Markt mit über 80 \% Marktanteil im Nahrungsmittelservicebereich. Sie beschäftigen insgesamt über 300.000 Angestellte. Diese relativ hohe Konzentration in einem ansonsten sehr diversifizierten Markt bietet sich für eine umfassende Organisierung an.

Die Kooperation ist in vielerlei Hinsicht interessant. SEIU und UNITE-HERE verstehen sich beide als organizing union, verwenden jedoch sehr unterschiedliche Organisierungsstrategien. Während die SEIU stark auf den Einsatz von professionellen Organizern setzt, verfolgt UNITEHERE einen eher partizipatorischen, zeitintensiveren Ansatz, ehrenamtliche Mitglieder zu Organizern zu trainieren und zu stärken. Deshalb erfordert das gemeinsame Organisierungsprojekt eine nicht $\mathrm{zu}$ unterschätzende Koordinierungsleistung.

Bereits wenige Monate nach Beginn der Kampagne (2004) konnten beide Gewerkschaften erste Abkommen mit den „Big 3 “ abschließen, die den Gewerkschaften Organisierungsrechte über 40.000 Beschäftigte in den folgenden vier Jahren zusprachen. Allerdings waren diese Verträge sehr schwach, gaben den Gewerkschaften wenig Erzwingungsoptionen und verpflichteten sie zur Friedenspflicht, was den Unternehmen auf regionaler Ebene keinen Anreiz gab, die Verträge einzuhalten. Dieser Verlauf hat innerhalb der Gewerkschaften, auch im Hinblick auf ähnliche Ansätze, die Diskussion aufgeworfen, ob ein schneller aber schlechter Vertrag besser ist als kein Vertrag und wie mit schlechten Verträgen umgegangen werden sollte (Szerlag/Weiner 2007).

\subsection{NATIONAL CLIENTBASED CAMPAIGNS}

Kundenorientierte Kampagnen orientieren sich an der JfJ-Strategie, auftraggebende Unternehmen auf die ausschließliche Nutzung von gewerkschaftlichen Reinigungsund Sicherheitsfirmen zu verpflichten. Diese Strategie ist sinnvoll, wenn es eine hohe Konzentration von nationalen Auftraggebern gibt, die eine Vielzahl von Reinigungsfirmen beschäftigen. Vorteil für die Gewerkschaft ist in einer solchen Konstellation eine geringe Anzahl von "Zielen“ (Auftraggeber), mit denen aber eine große Anzahl von Reinigungsfirmen erreicht werden kann. Bei dieser urgewerkschaftlichen Strategie, den Arbeitsmarkt kontrollieren zu wollen, fungiert die Gewerkschaft quasi als Vermittlungsagent für die Dienstleistungsunternehmen, da nach der Unterzeichung des Master-Abkommens nicht gewerkschaftliche Unternehmen aus dem Markt gedrängt werden. ${ }^{13}$ Diese Strategie wird seit 2004 in der „Justice for Mallworkers"-Kampagne bei drei großen Real Estate Investment Trusts (REIT), die Einkaufszentren bauen und unterhalten, verfolgt. Im Zuge dieser Kampagne werden neue, unternehmensorientierte Taktiken gegen REITs verwendet; beispielsweise werden Aktienanteile der Unternehmen gekauft, um die Aktionärsversammlungen als Öffentlichkeitsplattform zu nutzen, es wird Druck über Investoren und Anleger ausgeübt und es werden zukünftige Expansionsprojekte der Unternehmen behindert, um das Unternehmen zum Einlenken zu bewegen. Nach Abschluss der „Justice for Mallworkers"-Kampagne ist eine Kampagne gegen nationale Fluglinien angedacht, da diese sich aufgrund ihrer Konzentration, ihrer geografischen Verteilung und ihrer Angreifbarkeit als strategisch günstige Ziele anbieten.

\subsection{NATIONAL CONTRACTOR CAMPAIGNS}

Arbeitgeberorientierte Kampagnen setzen genau umgekehrt an und zielen auf umfassende Verträge mit national operierenden Dienstleistungsunternehmen, ähnlich einem Firmentarifvertrag. Da die Gebäudedienstleistungsindustrie sich zunehmend zentralisiert, gibt es eine Reihe von Unternehmen, auf die die SEIU an unterschiedlichen Orten immer wieder trifft. Dies bietet die Chance mit einem nationalen Abkommen Zehntausende von Mitgliedern zu gewinnen, die bestehende „Marktmacht" auszubauen und zugleich Zugang zu neuen „Märkten“ zu bekommen.

2006 konnte die SEIU als Ergebnis einer solchen Kampagne mit dem Serviceunternehmen Unicco einen ersten nationalen Vertrag abschließen, der der SEIU breite Anerkennungsrechte zusichert und der als Vorbild für künftige Abkommen gilt. Dieser Ansatz birgt auch Konflikte und zwar dann, wenn Unternehmen anvisiert werden, mit denen bereits örtliche Vereinbarungen bestehen. Seit 2007 wird beispielsweise eine Kampagne gegen das nationale Reinigungsunternehmen ABM geführt, mit dem die SEIU bereits eine Reihe von Verträgen abgeschlossen hat. ABM hat gedroht, diese Verträge aufzukündigen, wenn die Gewerkschaft ihre Kampagne nicht einstellt. Dennoch ist es laut SEIU nur eine Frage der Zeit, bis dieser Ansatz eine zentralere Rolle einnimmt, weil er bislang die einzige Lösung darstellt, ein Unternehmen nicht wieder und wieder konfrontieren zu müssen (Knaus 2007).

\subsection{NEW MARKET CAMPAIGNS}

Der Zugewinn von neuen Märkten, das heißt, die geografische Ausweitung auf unorganisierte Regionen, ist die am ehesten am traditionellen JfJ-Modell ausgerichtete Strategie und wird bislang am breitesten praktiziert. Im Jahr 2000 beschloss die SEIU erstmalig „Durchbruch-Kampagnen“ (Breakthrough Organizing Campaigns) in bislang völlig unerschlossenen Regionen in Right-to-Work-Staaten im Süden der USA, um die großen Verankerungslücken zwischen den Küstenregionen in Ost und West zu schließen. Die Wahl der Ziele fiel auf Miami, Houston und die sogenannten TriCities Indianapolis, Cincinnati und Columbus. Sie liegen geografisch günstig, es operierten dort überwiegend nationale Unternehmen, mit denen die SEIU anderorts Verbindungen hatte und die Arbeitsbedingungen waren vergleichsweise schlecht. Nach dem Beschluss "adoptierten“ die Locals jeweils eins der drei Ziele und koordinierten die Kampagnen, die aufgrund der verschiedenen lokalen Umstände sehr unterschiedlich verliefen. Während die SEIU an der Universität in Miami nur einen sehr schwachen ersten Vertrag abschließen konnte, wurde JfJ in Houston zur größten, längsten und spektakulärsten gewerkschaftlichen Organisierungskampagne in

12 Vgl. Website: http://www.serviceworkersunited. org, Download vom 16.10.2007.

13 Hierfür baut die SEIU einen sogenannten Trigger (Anreiz) in die Verhandlungen mit ein, in dem sie sich bereit erklärt, die Tarifverhandlungen nicht eher zu beginnen, bis mindestens $65 \%$ der Arbeitgeber organisiert sind, wofür sie im Gegenzug ungehinderte Organisierungsrechte einfordert. 
der Privatwirtschaft, die jemals im Süden der USA stattgefunden hat (Franklin 2006). Der Durchbruch von Jff in Houston hatte große Symbolwirkung für die Gewerkschaft und auch Auswirkungen auf andere JfJ-Kampagnen, die im Anschluss schneller abgeschlossen werden konnten, wenn es sich um dieselben Unternehmen handelte (Sixel 2006). Auch die Tri-Cities Kampagne ist mittlerweile abgeschlossen.

Der Zugewinn von neuen Märkten gilt als sehr erprobt und sinnvoll, um eine bundesweite Präsenz aufzubauen, nimmt aber enorme zeitliche und finanzielle Ressourcen in Anspruch. Die Houston Kampagne hat die SEIU weit über 3 Mio. Dollar gekostet und verursacht nach wie vor beträchtliche Kosten, da das dortige Local weiterhin für einige Jahre subventioniert werden muss, bis die Mitgliederbeiträge die Kosten tragen. Insgesamt nahm die Houston Kampagne nahezu sechs Jahre in Anspruch. Das jahrelange "Ausharren“ bis zum Sieg ist mittlerweile ein „Markenzeichen“ von Jfj und erfüllt die Signalfunktion, dass nicht nachgegeben wird, ehe ein Erfolg erzielt ist (Schwartz 2007). Von den acht größten Büromärkten in den USA hat die SEIU nun mittlerweile sechs organisiert, lediglich Dallas und Atlanta stehen noch aus, allerdings ist aus Ressourcengründen nicht zu erwarten, dass dort ähnlich umfassende Kampagnen durchgeführt werden.

\section{Fazit: Wachstum statt Organisierung}

Betrachtet man die oben beschriebenen Wachstumskonzepte der SEIU, fallen mehrere Punkte ins Auge, die einen Trend andeuten. Nahezu alle Kampagnen orientieren sich an nationalen Zielen, seien es Arbeit- oder Auftraggeber, was eine Kooperation der landesweit verteilten Locals der Gebäudedienstleistungen erfordert und tendenziell deren Fusion zuarbeitet. Zentralisierte Locals werden von der SEIUFührung favorisiert, da sie vermeintlich eher in der Lage seien, wirkliche Marktmacht auszuüben. Diese Prioritätensetzung ist jedoch in der Gewerkschaft umstritten, da große Locals als weitaus weniger demokratisch gelten als kleine. Kleine Locals bieten mehr Partizipationsmöglichkeiten für Mitglieder, da die Hierarchien flacher und die geografischen Grenzen enger gesetzt sind. Das „Superlocal“ SEIU 32BJ beispielsweise, ehemals basiert in $\mathrm{New}$ York City, erstreckt sich mittlerweile über sieben Staaten an der Ostküste und vereint Connecticut, Washington D. C., Pennsylvania, Maryland, New Jersey und Teile von Virginia. Die vormals unabhängigen Locals hatten eigene Vorstandsstrukturen und Vorsitzende, jetzt sind sie alle dem 32BJ Präsidenten Mike Fishman unterstellt und haben gegenüber der früheren Struktur in New York City weitaus weniger Einfluss.

Auch ist festzustellen, dass Unternehmenskampagnen mit geringer oder keiner Beteiligung der Beschäftigten zunehmen und einen größeren Stellenwert in der Konzeption von Kampagnen einnehmen, da sie kostengünstiger und allein gestützt auf den hauptamtlichen Apparat durchzuführen sind. Es bliebe allerdings noch zu untersuchen, inwieweit Corporate Campagins auch dieselben Resultate wie Comprehensive Campaigns erzielen.

Eine weitere Auffälligkeit ist die Koexistenz von Konfliktorientierung und Sozialpartnerschaft in den oben beschriebenen Organizing-Modellen - auf die andere Autoren bereits hingewiesen haben (Dribbusch 2007; Frege/Kelly 2004). Die in Deutschland militant erscheinenden Kampagnen gründen in der gewerkschaftsfeindlichen Haltung vieler US-Unternehmen, die Gewerkschaft ist aber gleichwohl, wann immer möglich, an kooperativen Beziehungen interessiert. Dies zeigen nicht nur wiederholte Appelle für den Aufbau von sozialpartnerschaftlichen Beziehungen seitens des Gewerkschaftsvorsitzenden Andy Stern (2006), sondern auch entsprechende Abkommen mit einigen Unternehmen im Gesundheitssektor, bei denen die Gewerkschaft zu einigen umstrittenen $\mathrm{Zu}$ geständnissen bereit war (Dribbusch 2007; Kirkland 2006; Stahl 2006). Die Parallelität von konfliktorientierten Kampagnen und Verträgen mit Einwilligung zur Friedenspflicht oder protektionistischen trigger-Abkommen birgt gleichwohl Spannungen, die eventuell auch innergewerkschaftliche Klärungen erfordern.

Was die SEIU für andere Gewerkschaften besonders interessant macht, ist jedoch nicht nur die ausgesprochen strategische Planung des Organisationswachstums, sondern das sind vor allem auch ihre Erfolge. Die SEIU zeigt, dass gewerkschaftliches Wachstum auch unter schwierigen Rahmenbedingungen planbar und möglich ist. Dabei sieht es die Gewerkschaft als Vorteil an, in Dienstleistungsbranchen zu organisieren, deren angebotene Arbeitsleistungen ortsgebunden und deshalb nicht verlagerbar sind. Allerdings hat mittlerweile der Begriff Wachstum (Growth) auch im Sprachgebrauch der SEIU den Begriff der Organisierung (Organizing) weitgehend ersetzt, obwohl er keinesfalls dasselbe meint. Während Organisierung auch einen Prozess der Organisationswerdung (union building) meint, begrenzt sich Wachstum auf die reine Addition von Mitgliedern.

Angesichts der extrem niedrigen gewerkschaftlichen Organisationsgrade in den USA $^{14}$ ist diese strikte Ausrichtung verständlich. Sie erscheint in den USA, wo Gewerkschaften institutionell nahezu gar nicht abgesichert sind, überlebensnotwendig. Die Fixierung der SEIU auf das Organisationswachstum steht jedoch im Widerspruch zu der teilweise verklärenden Vorstellung einer bewegungsorientierten Social Justice-Gewerkschaft. Diese Ambivalenz zieht sich durch die schillernde Geschichte von Justice for Janitors wie durch ihre sonstige Organisierungspraxis. Ihren Ruf als radikale, soziale Bewegung erlangte die Kampagne durch die Anwendung von militanten Aktionsformen, die Einbindung von Bewegungsaktivisten und migrantischen Beschäftigten im Niedriglohnsektor. Dabei täuschte die radikale Rhetorik darüber hinweg, dass es jenseits der Mitgliedergewinnung kaum übergeordnete Ziele, wie soziale Gerechtigkeit oder gesellschaftlichen Wandel, gab. JfJ war von Beginn an eine strategische top-down Mitgliedergewinnungskampagne (Milkman/Wong 2001; Savage 2006; Waldinger 1998), und daher ist es nicht verwunderlich, dass oben beschriebene Erneuerungsstrategien von JfJ diese Linie konsequent fortführen. Dies steht jedoch nicht im Widerspruch zu tatsächlichen Emanzipationsbewegungen, die diese Kampagne angestoßen hat und die zum Beispiel die Mobilisierung von migrantischen Beschäftigten betreffen oder das immigrationspolitische Engagement der SEIU.

\footnotetext{
4 Für 2006 liegt der allgemeine gewerkschaftliche Organisationsgrad bei ca. $12 \%$; in der Privatwirtschaft liegt er bei ca. 7,4 \%. Nach Sektoren aufgeschlüsselt, schwankt der Organisationsgrad erheblich: Während der öffentliche Sektor mit 36 \% sehr gut organisiert ist, ist der private Dienstleistungssektor mit ca. $2 \%$ weitgehend unorganisiert. Alle Angaben: Unionstats.com: http://www. unionstats.com, 17.10.2007.
} 
Geht es um gewerkschaftliche Reformkonzepte, sollten die SEIU und JfJ nicht verklärt werden, sondern mit dem nötigen interessiert kritischen Blick betrachtet werden. Dass eine Eins-zu-Eins-Übertragung des JfJ-Organisierungsmodells auf deutsche Gewerkschaften weder möglich noch sinnvoll ist, darauf haben bereits mehrere Autoren hingewiesen, ebenso aber auch auf die Möglichkeit, einzelne Strategien zu adaptieren und weiterzuentwickeln (Dribbusch 2007; Frege 2000). Dies gilt auch für diesen Beitrag. Da deutsche Gewerkschaften erst allmählich beginnen, Strategien zur
Überwindung der Mitgliederkrise zu entwickeln, könnte jedoch nicht nur der Organizing-Aspekt von Justice for Janitors, der derzeit in deutschen Gewerkschaften intensiv diskutiert wird, sondern insbesondere auch das strategische Vorgehen der SEIU einen anregenden Impuls darstellen.

\section{LITERATUR}

Bremme, P./Fürniß, U./Meinecke, U. (Hrsg.) (2007): Never work alone. Organizing - ein Zukunftsmodell für Gewerkschaften, Hamburg Brinkmann, U./Choi, H./Detje, R./Dörre, K./Holst. H./Karakayali, S./ Schmalstieg, C. (2008): Strategic Unionism: Aus der Krise zur Erneuerung? Umrisse eines Forschungsprogramms, Wiesbaden

Bronfenbrenner, K. (1994): Employer behavior in certification elections and first contract campaigns: implications for labor law reform, in: Friedman, S./Hurd, R./Oswald, R./Seeber, R. (Hrsg.): Restoring the Promise of American Labor Law, Ithaca, S. 75-89

Bronfenbrenner, K. (2001): Changing to Organize, in: The Nation, September 3

Bronfenbrenner, K./Juravich, T./Friedman, S./Hurd, R./Oswald, R./

Seeber, R. (Hrsg.) (1998): Organizing to Win: New Research on Union Strategies, Ithaca

Bronfenbrenner, K./Hickey, R. (2003): Winning is Possible, in: Multinational Monitor 6, June, online verfügbar unter: http://www. multinationalmonitor.org/mm2003/03june/june03corp1.html, 16.10.2007 Bronfenbrenner, K./Hickey, R. (2004): Changing to Organize. A National Assessment of Union Strategies, in: Milkman, R./Voss, K. (Hrsg.): Rebuilding Labor. Organizing and Organizers in the New Union Movement, Ithaca, S. 17-61

Casey-Bolaños, M. (1999): Justice for Janitors: The SEIU's Campaign to Raise Standards for Contract Janitors, working paper

Choi, H. (2008): Know your enemy. Strategische Unternehmensrecherche als wesentliches Element in gewerkschaftlichen Organizingkampagnen in den USA, in: Kampf um Teilhabe: Akteure, Orte, Strategien, Promovierendentagung der Hans-Böckler-Stiftung 2007, im Erscheinen Dribbusch, H. (1998): Mitgliedergewinnung durch offensive Interessenvertretung: Neue gewerkschaftliche Organisierungsansätze aus den USA - Das Beispiel "Justice for Janitors", in: WSI-Mitteilungen 5, S. 281-291 Dribbusch, H. (2003): Gewerkschaftliche Mitgliedergewinnung im Dienstleistungssektor. Ein Drei-Länder-Vergleich im Einzelhandel, Berlin Dribbusch, H. (2007): Das ,Organizing-Modell'. Entwicklung, Varianten, Umsetzung, in: Bremme, P./Fürniß, U./Meinecke, U. (Hrsg.): Never work alone. Organizing - ein Zukunftsmodell für Gewerkschaften, Hamburg, S. 24-52

Erickson, C./Fisk, C./Milkman, R./Mitchell, D. J. B./Wong, K. (2007):

Unions and Low-Wage Immigrant Workers: Lessons from the Justice for Janitors Campaign in Los Angeles, 1990-2002, revised 2007, in: http://www.law.berkeley.edu/institutes/csls/Fisk\%20paper.doc, 28.3.2007
Fantasia, R./Voss, K. (2004): Hard Work. Remaking the American Labor Movement, Berkeley

Fairbrother, P./Yates, C. A. B. (2003): Trade unions in renewal. A Comparative Study, London/New York

Fletcher, B./Hurd, R. (1998): Beyond the Organizing Modell: The Transformation Process in Local Unions, in: Bronfenbrenner, K./Juravich, T./Friedman, S./Hurd, R./Oswald, R./Seeber, R. (Hrsg.) (1998): Organizing to Win: New Research on Union Strategies, Ithaca, S. 37-53

Franklin, S. (2006): Chicago labor leader scores Houston coup, in: Chicago Tribune, 25. November, Business Section, Zone C, S. 1

Frege, C. (2000): Gewerkschaftsreformen in den USA: Eine kritische Analyse des 'Organisierungsmodells', in: Industrielle Beziehungen 3, S. 260280

Frege, C./Kelly, J. (Hrsg.) (2004): Varieties of Unionism. Strategies for Union Revitalization in a Globalizing Economy, Oxford Greer, I. (2006): Business union vs. business union? Understanding the split in the US labour movement, in: Capital \& Class 3, S. 1-6

Hurd, R./Milkman, R./Turner, L. (2003): Reviving the American labour movement: Institutions and Mobilization, discussion paper DP/132/2002, International Institute for Labour Studies, Geneva

Katz, H/Kochan, T. (2003): An Introduction to Collective Bargaining \& Industrial Relations, New York

Knaus, H. (2007): Toward a National ABM Agreement (National Contractor Approach), Präsentation auf der SEIU National Researcher Conference, 1.-3 Oktober in Adelphi, Maryland

Kirkland, R. (2006): The New Face of Labor, in: Fortune Magazine 10, online verfügbar unter: http://money.cnn.com/magazines/fortune/ fortune_archive/2006/10/16/8390290/index.htm, 31.10.2007 Knowles, F. (2007): 2 years after labor split, Change to Win reflects, in: Chicago Sun Times, 24.09.

Lerner, S. (2001): Organizing Strategies for the 21st Century, Präsentation auf "The New Economy and Union Responses"-Konferenz, University of California, Institute for Labor and Employment, Los Angeles, 9. März

Levine, A. (2007): Unionbusting Confidential. To keep out organized labor, you need the union-busting law firm Jackson Lewis, in: In These Times, September 2006, http://www.inthesetimes.com/article/3326/ unionbusting_confidential/, 16.10.2007.

Logan, J. (2006): The Union Avoidance Industry in the United States, in: British Journal of Industrial Relations 4, S. 651-675 
Long, V. (2007): SEIU Planning Process, Präsentation auf der SEIU National Researcher Conference 2007, 1.-3 Oktober in Adelphi, Maryland Lopez, S. H. (2004): Reorganizing the Rust Belt. An Inside Study of the American Labor Movement, Berkeley

Manheim, J. B. (2005): Trends in Union Corporate Campaigns, A Briefing Book, Washington

Milkman, R. (2006a): Organizing the Unorganizable, in: Boston Review September/October, online verfügbar unter: http://bostonreview. net/BR31.5/milkman.html, 16.10.2007

Milkman, R. (2006b): L. A. Story. Immigrant Workers and the Future of the U. S. Labor Movement, New York

Milkman, R./Wong, K. (2001): Organizing Immigrant Workers, in: Turner, L./Katz, H./Hurd, R.: Rekindling the Movement. Labor's quest for relevance in the $21^{\text {st }}$ century, Ithaca/London, S. 99-128

Milkman, R./Voss, K. (2004): Rebuilding Labor: Organizing and Organizers in the New Union Movement. Ithaca/London

Pastreich, M. (2007): Developing a Plan for Property Services, Präsentation auf der SEIU National Researcher Conference 2007, 1.-3 Oktober in Adelphi, Maryland

Savage, L. (2006): Justice for Janitors: Scales of Organizing and Representing Workers, in: Antipode 3, S. 645-666

Schwartz, C. (2007): Interview über Justice for Janitors in Houston, 2. Oktober, Adelphi

SEIU (2003): SEIU members vote yes on plan to build stronger union, Press Release June 17
SEIU (2007): Houston Janitors Website http://www.houstonjanitors.org/ how-it-works/, 16.10.2007

Sixel, L. M. (2006): 'A foothold into the South': Janitor Strike, in: Houston Chronicle, 14. November, Business and Financial News, S. 1

Stahl, L. (2006): Andy Stern: The New Boss. CBS News, 60 Minutes, 14. Mai. Online verfügbar unter: http://www.cbsnews.com/stories/2006/ 05/12/60minutes/main1614451 page2.shtml, 31.10.2007

Stern, A. (2006): A Country that Works. Getting America Back on Track, New York

Szerlag, H./Weiner, W. (2007): Expanding into Multi-Service, Präsentation auf der SEIU National Researcher Conference, 1.-3 Oktober 2007 in Adelphi, Maryland

Turner, L./Hurd, R. (2001): Building Social Movement Unionism, in: Turner, L./Katz, H./Hurd, R.: Rekindling the Movement. Labor's quest for relevance in the $21^{\text {st }}$ century, Ithaca/London, S. 9-26

Turner, L./Cornfield, D. B. (2007): Labor in the New Urban Battlegrounds, Ithaca

Voss, K./Rachel S. (2000): Breaking the Iron Law of Oligarchy: Union Revitalization in the American Labor Movement, in: American Journal of Sociology 2, S. 303-349

Waldinger, R. D./Erickson, C./Milkman, R./Mitchell, D./ Valenzuela, A./Wong, K./Zeitlan, M. (1998): Helots No More: A Case Study of the Justice for Janitors Campaign in Los Angeles, in: Bronfenbrenner, K./ Oswald, R./Friedman, S./Seeber, R./Hurd, R. (Hrsg.): Organizing to Win: New Research on Union Strategies, Ithaca, S. 102-119 